\title{
The diagonalized contrast source approach: an inversion method beyond the Born approximation
}

\author{
Aria Abubakar ${ }^{1}$, Tarek M Habashy ${ }^{1}$, Peter M van den Berg ${ }^{2}$ \\ and Dries Gisolf ${ }^{2}$ \\ ${ }^{1}$ Schlumberger-Doll Research 36, Old Quarry Road, Ridgefield, CT-06877, USA \\ ${ }^{2}$ Faculty of Applied Sciences, Delft University of Technology, 2628 CJ Delft, The Netherlands \\ E-mail: aabubakar@ridgefield.oilfield.slb.com
}

Received 10 November 2004

Published 23 February 2005

Online at stacks.iop.org/IP/21/685

\begin{abstract}
This paper deals with the imaging and inversion of the constitutive material properties of bounded objects embedded in a known background medium. The inversion utilizes measurements of the scattered field due to the illumination of the objects by a set of known single-frequency wave fields. We present two inverse scattering methods that approximately recast the full nonlinear inversion into a number of linear inversion steps. The two methods are as computationally efficient as the constrained Born inversion but provide reconstruction results that are far superior and are almost comparable in quality to the ones obtained using a full nonlinear iterative inversion. The linear inversion steps follow what is referred to as the source-type integral equation approach in which the contrast sources are inverted for in the first step from the linear data equation. In this step, we employ a local (diagonal) approximation of the operator that relates the contrast sources to the incident field. Once the contrast sources have been determined, the total internal wave fields follow from a direct application of the object equation. In the third and final step, the contrast function is estimated from either the constitutive relation (first method) or from solving the data equation again, this time in terms of the contrast profile (second method). The computational cost required by the first method is comparable to that of a constrained Born inversion. With only twice the computational cost, the second method invariably gives an almost perfect image. We will demonstrate the two methods for a number of representative synthetic examples, both in two and three dimensions.
\end{abstract}

(Some figures in this article are in colour only in the electronic version)

\section{Introduction}

A large class of inverse problems deal with the determination of the constitutive material parameters of bounded objects embedded in a known background medium. The inversion 
utilizes data from measurements of the scattered field when the object is illuminated with a known single-frequency wave field. The unknown parameter to be inverted for is usually the index of refraction which may be complex if the medium is lossy. This class of problems is usually addressed by employing the domain integral equation which governs the wave phenomenon within the object. An extensive review of linear and nonlinear scalar inverse scattering algorithms based on the so-called Lippmann-Schwinger integral equation can be found in Colton et al [9], Habashy and Mittra [10], Lesselier and Duchene [15], Sabatier [21] and van den Berg [28]. Recent publications on methods that approach these problems using differential equation formulations can be found in $\mathrm{Bao}$ and $\mathrm{Li}[3]$ and Bulyshev et al [22].

In the Born approximation, the total field inside the scattering object is replaced by the incident field present in the embedding medium. This approximation is effective in cases when the material contrast is small and as long as the size of the object is not too large relative to the wavelength (Born [6], Born and Wolf [7], Nieto-Vesperinas [19]). Recently, and for scalar waves, an error bound for the Born approximation has been derived by Natterer [18]. In the inverse scattering problem, the Born approximation is often used as a starting point, since it renders the problem linear with respect to the material contrast.

Related to the Born approximation is the Rytov approximation, see Oristaglio [20], which assumes a particular functional form for the field in terms of the exponential of a complex phase function. The Rytov approximation or transformation is widely used in problems where the length scale of fluctuation is large compared to the wavelength, since its exponential representation is thought to be more representative of wave propagation.

A significant improvement in the Born approximation can be achieved by employing what is referred to as the extended Born approximation by Habashy and co-workers (see e.g. [11] and [25]). The computation cost required by this extended Born approximation is comparable to the original Born approximation; however, the nonlinear nature of this approximation allows one to obtain accurate numerical simulations for a larger range of material contrasts and object sizes. For a low-frequency inversion problem, a two-step procedure based on the extended Born approximation was introduced by Torres-Verdin and Habashy [24]. Both steps involve the solution of a linear problem. In the first step a first kind integral equation (with respect to the data) has to be solved, while in the second step a second kind integral equation for the object contrast has to be inverted.

In this paper, we introduce a new three-step procedure which introduces a localized approximation of the scattering operator without enforcing an explicit form for it as was done in [24]. The linear inversion steps follow what is referred to as the source-type integral equation approach, see Habashy et al [12], in which the contrast sources are inverted for in the first step from the linear data equation. Once the contrast sources have been determined, the total internal wave fields follow from a direct application of the object equation. In the third and final step, the contrast function is estimated using one of two approaches. In the first approach (referred to as the diagonalized contrast source method I, abbreviated as DCSI-I) the contrast function is estimated directly from the constitutive relation. Whereas in the second approach (referred to as the diagonalized contrast source method II, abbreviated as DCSI-II) the contrast function is estimated by solving the data equation again, this time in terms of the contrast profile. What makes such approaches feasible is the introduction of a diagonalization approximation of the full contrast operator (similar to the concept in Habashy et al [11]). This allows the source-type integral equation approach with its three linear steps to be implemented in a robust manner, in which the nonuniqueness encountered in the reconstruction of the contrast sources in the first step is alleviated by the imposition of the diagonal form constraint on the contrast operator. 
Two additional enabling methods are introduced to guarantee the robustness and convergence of the above approach. The first method is introduced in the implementation of the first step (of inverting the contrast sources) where we have applied the contrast source inversion of Abubakar et al [1]. The second enabling method is introduced in the implementation of the third step (of inverting the contrast profile) where we have applied the multiplicative regularized inversion of [1].

We will demonstrate the two methods by showing reconstruction results for some representative synthetic examples both in two and three dimensions.

\section{The inverse problem}

Let $D$ denote the interior of a bounded domain with piecewise smoothly discontinuous interfaces. We assume that an unknown scatterer, $B$, of arbitrary cross section is contained within this domain $D$. This constitutes a priori information on the approximate location of the scattering object. The domain $D$ may have to be chosen sufficiently large to ensure inclusion of $B$, but this will incur a computational cost.

A Cartesian coordinate system is centred at $D$. Spatial points are denoted by $\boldsymbol{p}=$ $\left(x_{p}, y_{p}, z_{p}\right)$ and $\boldsymbol{q}=\left(x_{q}, y_{q}, z_{q}\right)$.

We restrict ourselves to the scalar field problem where the scattering object is irradiated successively by a number of known time-harmonic incident fields $u_{j}^{\text {inc }}(\boldsymbol{p}), j=1, \ldots, J$, originating from different source positions. The time convention used in this paper is $\exp (-\mathrm{i} \omega t)$, where $\omega$ is the angular frequency of the incident field.

For each incident field, the total field $u_{j}(\boldsymbol{p})$ in the domain $D$ and the scattered field exterior to $D$ satisfy the following integral equations:

$$
\begin{aligned}
& u_{j}(\boldsymbol{p})=u_{j}^{\mathrm{inc}}(\boldsymbol{p})+\int_{D} g(\boldsymbol{p}, \boldsymbol{q}) \chi(\boldsymbol{q}) u_{j}(\boldsymbol{q}) \mathrm{d} v(\boldsymbol{q}), \quad \boldsymbol{p} \in D, \\
& u_{j}^{\mathrm{sct}}(\boldsymbol{p})=\int_{D} g(\boldsymbol{p}, \boldsymbol{q}) \chi(\boldsymbol{q}) u_{j}(\boldsymbol{q}) \mathrm{d} v(\boldsymbol{q}), \quad \boldsymbol{p} \notin D,
\end{aligned}
$$

where $g(\boldsymbol{p}, \boldsymbol{q})$ is the Green function in the embedding medium. Apart from a factor of $k_{0}^{2}, g(\boldsymbol{p}, \boldsymbol{q})$ represents the field response at an observation point $\boldsymbol{p}$ due to a point source located at $\boldsymbol{q}$. Further, $\chi(\boldsymbol{q})=k^{2}(\boldsymbol{q}) / k_{0}^{2}(\boldsymbol{q})-1$ denotes the contrast of the scattering object with respect to its embedding medium, while $k(\boldsymbol{q})$ and $k_{0}(\boldsymbol{q})$ denote the wavenumbers of the scattering object and the embedding, respectively.

In the inverse scattering problem, the scattered field $f_{j}=u_{j}^{\text {sct }}$ will be measured on some surface $S$ exterior to $D$. The integral representation (2) for points exterior to $D$, written symbolically as

$$
f_{j}=G_{S}\left\{\chi u_{j}\right\}, \quad \boldsymbol{p} \in S,
$$

refers to the data equation, while the integral equation (1), written symbolically as

$$
u_{j}^{\text {inc }}=u_{j}-G_{D}\left\{\chi u_{j}\right\}, \quad \boldsymbol{p} \in D,
$$

refers to the object equation. The operator $G_{S}$ is an operator mapping $L^{2}(D)$ onto $L^{2}(S)$ whereas $G_{D}$ is an operator mapping $L^{2}(D)$ onto $L^{2}(D)$. These operators are given by

$$
G_{S, D}\left\{w_{j}\right\}=\int_{D} g(\boldsymbol{p}, \boldsymbol{q}) w_{j}(\boldsymbol{q}) \mathrm{d} v(\boldsymbol{q}), \quad \boldsymbol{p} \in\{S, D\} .
$$

The subscripts $S$ and $D$ of the integral operator $G_{S, D}$ identify the location of the field point $\boldsymbol{p}$ in the space. Let us further denote the norm and inner product in $L^{2}(S)$ and $L^{2}(D)$ by 
appending a subscript $S$ or $D$ as appropriate, i.e. $\|\cdot\|_{D},\langle\cdot, \cdot\rangle_{D}$, etc. The norm and the inner product are defined as

$$
\left\|u_{j}\right\|_{D, S}=\left\langle u_{j}, u_{j}\right\rangle_{D, S}^{\frac{1}{2}}, \quad\left\langle u_{j}, v_{j}\right\rangle_{D, S}=\int_{D, S} u_{j}(\boldsymbol{p}) \bar{v}_{j}(\boldsymbol{p}) \mathrm{d} v(\boldsymbol{p}),
$$

where the overbar in $\bar{v}_{j}(p)$ denotes complex conjugation.

If the scattered field data, measured on $S$, is $f_{j}(\boldsymbol{p})$, which includes the measurement error, noise and any other signal contamination, then (3) will not, in general, be satisfied if $f_{j}$ replaces $u_{j}^{\text {sct }}$. In fact we use this data equation to define the discrepancy between the measured data and the predicted scattered field corresponding to $\chi$ and $u_{j}$ in $D$. The inverse scattering problem consists of determining $\chi(p)$ from knowledge of the incident field, $u_{j}^{\text {inc }}(\boldsymbol{p})$, on $D$ and the scattered field, $f_{j}(\boldsymbol{p})$, on $S$. In general this problem is both nonlinear and highly ill-posed. Using our definition of the norm, the inverse scattering problem is recast as the optimization problem of finding a function $\chi$ that minimizes the misfit in the data equation of (3), subject to the constraint that the object equation (4) is satisfied in some sense. The existence of a minimizer can be guaranteed by a suitable choice of the class of admissible values of $\chi$. However, whether this minimizer is related to a local or a global minimum will remain an open issue.

The formal solution of the object equation (4) is written as

$$
u_{j}=L^{-1}\left\{u_{j}^{\text {inc }}\right\}, \quad L=I-G_{D} \chi,
$$

where $I$ is the unit operator. Subsequently, (7) is substituted into the data equation (3), so that the data error becomes

$$
\rho_{j}=f_{j}-G_{S}\left\{\chi L^{-1}\left\{u_{j}^{\text {inc }}\right\}\right\} .
$$

The inverse problem is posed as determining an approximate contrast $\chi=\chi^{\text {appr }}$ from

$$
\chi^{\text {appr }}=\underset{\chi}{\arg \min } \frac{\sum_{j=1}^{J}\left\|\rho_{j}(\chi)\right\|_{S}^{2}}{\sum_{j=1}^{J}\left\|f_{j}\right\|_{S}^{2}} .
$$

Other normalizations of the cost functional are also possible (see for example [13]). This inverse problem is a classic example of an ill-posed problem and is treated by introducing Tykhonov regularizers to the cost functional. The inverse problem is nonlinear and the minimization of (9) can only be performed by an iterative updating of the contrast $\chi$, where one has to compute the operation $L^{-1} u_{j}^{\text {inc }}$ at each update, which, in fact, is a forward solution of (4).

\section{A localized approximation}

We arrive at a significant simplification of the inverse problem when we approximate the contrast operator $\chi L^{-1}$ by a diagonal one. To this end, we assume that there exists a function $\eta=\eta(p)$, independent of the excitations, $j$, such that

$$
\chi L^{-1}\left\{u_{j}^{\text {inc }}\right\} \approx \eta u_{j}^{\text {inc }}, \quad \text { for all } j
$$

where $\eta$ is defined as the approximate diagonal contrast operator. This approximation encompasses the Born approximation [6, 7], the extended Born approximation [11] and a modified version of the Rytov approximation [11]. 


\subsection{The Born approximation}

When the scatterer is small in size relative to the relevant length-scale of the problem (e.g., wavelength, skin depth, transmitter-receiver spacing, etc) or the index of refraction of the object relative to its embedding medium is close to unity (the so-called weak scatterers), then the well-known Born approximation consists of approximating the internal field, $u_{j}$ in $D$, to be the incident field. In fact, it is obtained by neglecting the term $G_{D}\left\{\chi u_{j}\right\}$ in (4) so that $u_{j} \approx u_{j}^{\text {inc }}$. Hence, with the Born approximation we have linearized the inverse problem.

With this approximation the data error becomes

$$
\rho_{j}^{\mathrm{B}}(\chi)=f_{j}-G_{S}\left\{\chi u_{j}^{\mathrm{inc}}\right\} \quad \text { on } S,
$$

and the inverse problem is posed as determining $\chi=\chi^{\mathrm{B}}$ as

$$
\chi^{\mathrm{B}}=\underset{\chi}{\arg \min } \frac{\sum_{j=1}^{J}\left\|\rho_{j}^{\mathrm{B}}(\chi)\right\|_{S}^{2}}{\sum_{j=1}^{J}\left\|f_{j}\right\|_{S}^{2}} .
$$

This optimization problem may be solved by adding a regularizer.

Comparing the expressions for the residual of the right-hand side of (8) and the one of the right-hand side of (11), we directly observe that in the Born approximation our diagonal approximation of the contrast operator of (10) is given by

$$
\eta=\chi
$$

\subsection{The extended Born approximation}

An improvement over the Born approximation is achieved in the extended Born approximation [11]. It results from an approximation of the internal field $u_{j}$ in the integral term of the object equation (4) by the first term of a Taylor expansion about the field point. This leads to an approximate object equation

$$
u_{j}^{\text {inc }}=u_{j}-u_{j} G_{D}\{\chi I\} \quad \text { on } \quad D
$$

where

$$
G_{D}\{\chi I\}=\int_{D} g(\boldsymbol{p}, \boldsymbol{q}) \chi(\boldsymbol{q}) \mathrm{d} v(\boldsymbol{q}) .
$$

The inverse problem is now posed as a nonlinear optimization problem to find $\chi$ as

$$
\chi^{\mathrm{eB}}=\underset{\chi}{\arg \min } \frac{\sum_{j=1}^{J}\left\|\rho_{j}^{\mathrm{eB}}(\chi)\right\|_{S}^{2}}{\sum_{j=1}^{J}\left\|f_{j}\right\|_{S}^{2}},
$$

where the residual is defined by

$$
\rho_{j}^{\mathrm{eB}}(\chi)=f_{j}-G_{S}\left\{\chi\left[1-G_{D}\{\chi I\}\right]^{-1} u_{j}^{\mathrm{inc}}\right\} .
$$

Note that in the right-hand side of this expression, the quantity $\left[1-G_{D}\{\chi I\}\right]^{-1}$ is not an inverse operator, but it denotes a reciprocal of a function. The optimization problem of (16) may be solved by adding a regularizer and it has been shown to be more effective than the Born approximation in a variety of applications, see Torres-Verdin and Habashy [23].

Comparing the expressions for the residual of the right-hand side of (8) and the one of the right-hand side of (17), we observe that this extended Born approximation is a more advanced diagonal approximation of the contrast operator of (10), namely,

$$
\eta=\chi\left[1-G_{D}\{\chi I\}\right]^{-1} \text {. }
$$

With the extended Born approximation, the problem is still nonlinear in the contrast $\chi$. However, using this extended Born approximation, a procedure employing two linear steps has been presented in Torres-Verdin and Habashy [24]. 


\section{The source-type integral equation}

In the rest of this paper, we introduce two inversion approaches that attempt to recast the nonlinear inversion into a sequence of linear inversion problems.

We observe that the data equation (3) contains both the unknown field and the unknown contrast in the form of a product,

$$
w_{j}(\boldsymbol{p})=\chi(\boldsymbol{p}) u_{j}(\boldsymbol{p}), \quad \boldsymbol{p} \in D,
$$

which can be considered as an equivalent source that produces the measured scattered field. We refer to this equivalent source as a contrast source. The data equation becomes

$$
f_{j}=G_{S}\left\{w_{j}\right\}, \quad \boldsymbol{p} \in S,
$$

while the object equation (4) becomes

$$
u_{j}=u_{j}^{\text {inc }}+G_{D}\left\{w_{j}\right\}, \quad \boldsymbol{p} \in D .
$$

At first glance, it appears that the easiest way to carry out the inversion is to solve first for the contrast sources, $w_{j}$, from the linear data equation (20). Once the contrast sources have been determined, the total wave fields, $u_{j}$ in $D$, follow simply from (21). Subsequently, the contrast function $\chi$ follows from the constitutive relation of (19) as the solution of the minimization problem [12]:

$$
\chi^{\mathrm{appr}}=\underset{\chi}{\arg \min } \sum_{j=1}^{J}\left\|w_{j}-\chi u_{j}\right\|_{D}^{2} .
$$

The minimizer can be represented explicitly as

$$
\chi^{\mathrm{appr}}(\boldsymbol{p})=\frac{\sum_{j=1}^{J} w_{j}(\boldsymbol{p}) \bar{u}_{j}(\boldsymbol{p})}{\sum_{j=1}^{J}\left|u_{j}(\boldsymbol{p})\right|^{2}} .
$$

This approach is referred to as the source-type integral equation [12]. It was shown in [12] that the naive way of implementing the above three steps is defeated by the non-uniqueness of the inverse source problem of (20) (see e.g. [5]). There exist non-trivial solutions of the homogeneous form of the data equation (20). However, these can only be restored by fully solving the inverse scattering problem to the end. On the other hand, it has also been shown that the minimum norm solution of the data equation is not the appropriate physical solution. Nonetheless source-type equations similar to (20) have served as the foundation for many inversion procedures, e.g. [1, 2, 26].

One approach to alleviate the non-uniqueness encountered in the reconstruction of the contrast sources from the data equation is to introduce additional conditions or constraints to be satisfied by the inverted sources. One such condition is to search for the contrast sources $w_{j}$ such that their associated contrast function $\chi$ are independent of the incident wave fields. Another possible constraint is to invert for the contrast sources $w_{j}$ subject to a particular functional form. For example, in the Born approximation, the functional form imposed is $w_{j} \approx \chi u_{j}^{\text {inc }}$. In the next section we introduce two approaches for reconstructing the contrast sources subject to the constraint that the full contrast operator has a diagonal form as given by $(10)$.

\section{DCSI-I: diagonalized contrast source inversion-method I}

As we outlined in the previous section, our objective is to develop an inversion approach which attempts to transform the nonlinear inversion into a sequence of linear inversion problems. 
This can be achieved using the source-type integral equation approach by inverting for the contrast sources $w_{j}$ from the data equation subject to the constraint that the full contrast operator is dominated by a diagonal form given by (10). This can be affected by introducing a method based on the contrast source inversion, as implemented in section IV of [1]. Such a method does not require a priori knowledge and provides a stable inversion result through the introduction of the following two-term cost functional:

$$
F_{1}\left(w_{j}, \eta\right)=\frac{\sum_{j=1}^{J}\left\|f_{j}-G_{S}\left\{w_{j}\right\}\right\|_{S}^{2}}{\sum_{j=1}^{J}\left\|f_{j}\right\|_{S}^{2}}+\frac{\sum_{j=1}^{J} \| \eta u_{j}^{\mathrm{inc}}-\left.w_{j}\right|_{D} ^{2}}{\sum_{j=1}^{J}\left\|\eta u_{j}^{\text {inc }}\right\|_{D}^{2}} .
$$

The contrast sources $w_{j}$ and the diagonal operator approximation $\eta$ are estimated through the minimization of this functional. It has been shown in [1] that a robust solution is obtained by an alternating direction minimization scheme in which $w_{j}$ and $\eta$ are alternately updated resulting in a continuous reduction of the above cost function after each update (see also [12]). The contrast sources are updated by a conjugate gradient algorithm, in which an explicit update for $\eta$ is obtained after each step. This explicit update is given by

$$
\eta=\frac{\sum_{j=1}^{J} w_{j} \bar{u}_{j}^{\mathrm{inc}}}{\sum_{j=1}^{J}\left|u_{j}^{\mathrm{inc}}\right|^{2}} .
$$

The presence of the second term in the functional of (24) always yields a nonzero contribution to the gradient, so that any instabilities of the conjugate gradient method caused by 'almost' zero directions are avoided. After solving for $w_{j}=w_{j}^{\text {inv }}$, the total wave fields $u_{j}$ in $D$ are simply computed from (21):

$$
u_{j}^{\text {inv }}=u_{j}^{\text {inc }}+G_{D}\left\{w_{j}^{\text {inv }}\right\}, \quad \boldsymbol{p} \in D
$$

and the contrast function $\chi$ is reconstructed from (22):

$$
\chi=\frac{\sum_{j=1}^{J} w_{j}^{\mathrm{inv}} \bar{u}_{j}^{\mathrm{inv}}}{\sum_{j=1}^{J}\left|u_{j}^{\mathrm{inv}}\right|^{2}} .
$$

After the estimation of $w_{j}$ and $\eta$ by the iterative minimization of the cost functional (24), the extra computation time required to implement (26) and (27) is negligible. Note that the diagonal operator approximation $\eta$ plays the role of a regularization to the inversion of $w_{j}$ through the cost function defined in (24). As will be shown in the section on numerical examples, this approach yields a remarkable improvement in the reconstructed profile compared to the Born inversion. In the numerical examples, we refer to this algorithm as DCSI-I.

\section{DCSI-II: diagonalized contrast source inversion-method II}

A further significant improvement in DCSI-I can be affected by replacing the reconstruction step of (27) with the inversion of $\chi$ from the data equation (3) with the internal field $u_{j}$ given by the update of (26). Again, to circumvent the ill-conditioning of the data equation, we recast this inversion problem in terms of the simultaneous inversion of $\chi$ and $w_{j}$ from the data equation of (20) subject to enforcing the constitutive relationship of (19) inside the scatterer with the internal field given by the update of (26). This is implemented by minimizing the following cost functional:

$$
F_{2}\left(w_{j}, \chi\right)=\frac{\sum_{j=1}^{J}\left\|f_{j}-G_{S}\left\{w_{j}\right\}\right\|_{S}^{2}}{\sum_{j=1}^{J}\left\|f_{j}\right\|_{S}^{2}}+\frac{\sum_{j=1}^{J}\left\|\chi u_{j}^{\mathrm{inv}}-w_{j}\right\|_{D}^{2}}{\sum_{j=1}^{J}\left\|\chi u_{j}^{\text {inv }}\right\|_{D}^{2}} .
$$


Note that the total computational cost of this method is roughly double the cost of the DCSI-I method. Although the inclusion of the object equation in the second term of (28) serves as a physical regularization of the ill-conditioned data equation in the first term of (28), the inversion results for $\chi$ can be controlled by introducing an additional regularizer that can help in accounting for any a priori information known about the contrast profile or in imposing any constraints such as limiting the total spatial variation of the contrast. In view of the previous experience, we propose to use the multiplicative regularized inversion of section $\mathrm{V}$ of [1], where we search for a solution of the contrast sources $w_{j}$ and the contrast function $\chi$ by an iterative minimization of

$$
C_{n}\left(w_{j}, \chi\right)=F_{2}\left(w_{j}, \chi\right) F_{n}^{R}(\chi)
$$

where the first factor is the original cost functional of (28) at the $n$th iteration, while the second factor is the so-called weighted $L^{2}$-norm factor ([1]) namely,

$$
F_{n}^{R}(\chi)=\frac{1}{V} \int_{D} \frac{|\nabla \chi(\boldsymbol{p})|^{2}+\delta_{n}^{2}}{\left|\nabla \chi_{n-1}(\boldsymbol{p})\right|^{2}+\delta_{n}^{2}} \mathrm{~d} v(\boldsymbol{p}),
$$

where $V=\int_{D} \mathrm{~d} v(\boldsymbol{p})$ denotes the volume of the object domain $D, \nabla \chi$ denotes the gradient of the contrast, and $\chi_{n-1}$ denotes the value of the contrast at the previous iteration. For how to chose $\delta_{n}^{2}$, we refer to [1]. In our numerical examples we will refer to this algorithm as DCSI-II.

We note that in minimizing the cost function $F_{1}\left(w_{j}, \eta\right)$ of $(24)$, we could have also applied the above multiplicative regularization. However, in this minimization step we do not invert for the contrast function, instead we determine an approximate diagonal contrast operator $\eta$, and since we do not have any a priori information regarding $\eta$, it is therefore not advisable to regularize with respect to $\eta$.

It can be seen that by minimizing the cost function of (28), higher order scattering effects are being taken into consideration.

\section{Two-dimensional numerical examples}

For our numerical examples, the test domain $D$ consists of a square. The homogeneous embedding is chosen to be lossless and $\lambda$ denotes the wavelength in the embedding. The discrete form of the algorithm is obtained by dividing the test domain into sub-squares, assuming the contrast and fields to be piecewise constant. The incident fields are chosen to be excited by line sources parallel to the cylindrical axis of the scatterer. The sources and receivers are taken to be equally spaced on a measurement circle $S$. The measured data are simulated by solving the direct scattering problem with the help of a conjugate gradient method [27]. The discretization grid employed to solve this direct scattering problem is twice finer than the one used in solving the inverse problem. After generation of the synthetic data, $10 \%$ random white noise is added using the following procedure:

$$
f_{j}^{\text {noise }}=f_{j}\left[1+\zeta\left(\operatorname{ran}_{1}+\mathrm{i} \operatorname{ran}_{2}\right)\right]
$$

where $\operatorname{ran}_{1}$ and $\operatorname{ran}_{2}$ are two random numbers varying from -1 up to 1 , and $\zeta=0.1$ corresponding to the percentage of noise added. Unless stated otherwise we use the backpropagation technique to generate initial guesses in all of our examples. The details of these computations can be found in van den Berg and Kleinman [26].

The inversion process is terminated if one of the following stopping conditions is satisfied.

- The difference between the normalized data error $F_{n}^{S}$ defined as

$$
F_{n}^{S}=\frac{\sum_{j=1}^{J}\left\|f_{j}-G_{S}\left\{w_{j, n}\right\}\right\|_{S}^{2}}{\sum_{j=1}^{J}\left\|f_{j}\right\|_{S}^{2}}
$$


(a)

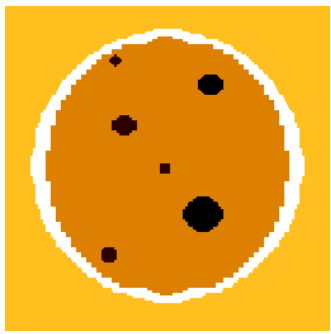

(b)

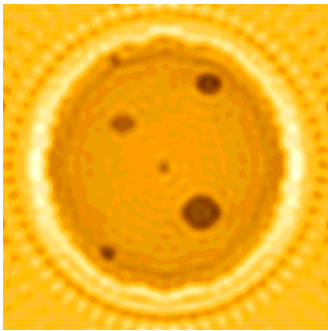

(c)

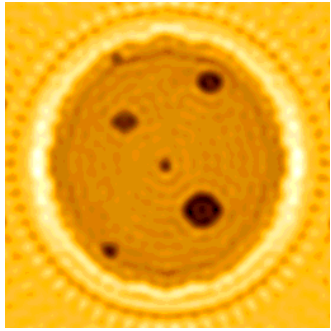

(d)

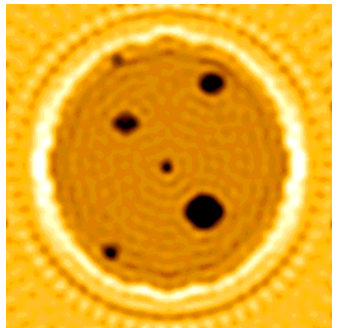

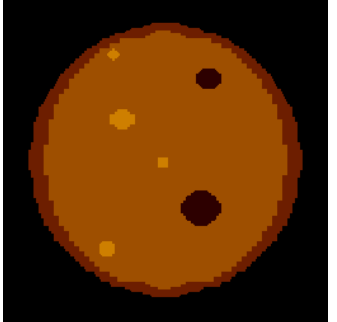
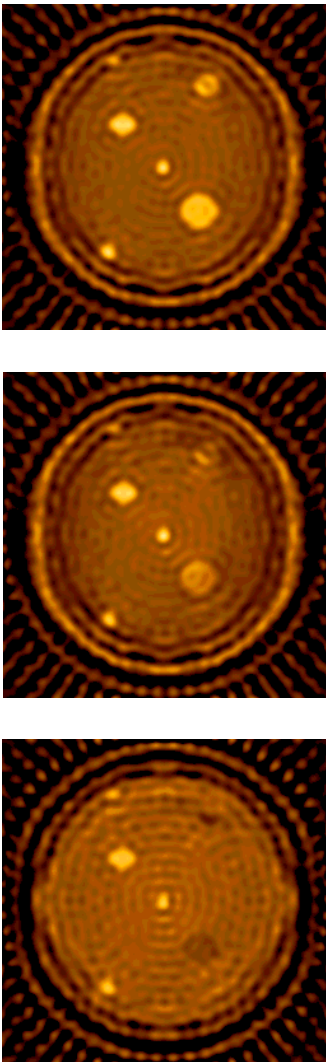

Figure 1. Inversion experiment of the acoustic tomography; left: real part of $\chi$, right: imaginary part of $\chi$; (a) true profile; (b) Born inversion; (c) DCSI-I and (d) DCSI-II.

at two successive iterates, $n$th and $(n-1)$-th, is within a prescribed tolerance factor, tole, of the normalized data error at the current iterate:

$$
\left|F_{n}^{S}-F_{n-1}^{S}\right| \leqslant \text { tole } \times F_{n}^{S} .
$$

- The total number of iterations exceeds a prescribed maximum $N_{\max }$.

\subsection{Example 1: acoustic tomography}

We start by showing the results of some reconstructions from acoustic data obtained from a computer generated breast phantom (Natterer [17]), which has been patterned after a phantom 
(a)

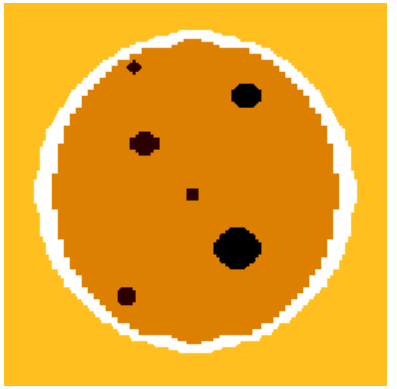

(b)

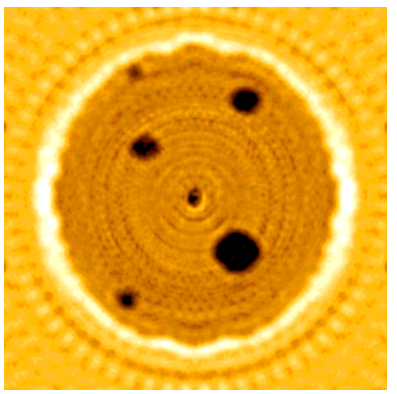

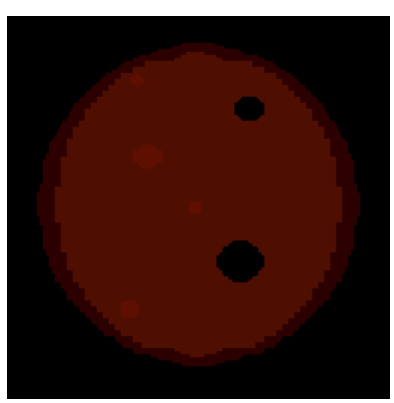

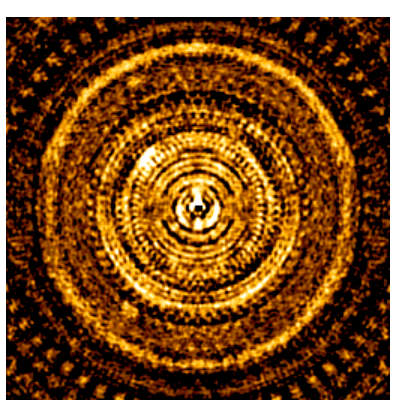

Figure 2. Inversion experiment of the acoustic tomography using $1 \mathrm{MHz}$ frequency of operation; left: real part of $\chi$, right: imaginary part of $\chi$; (a) true profile and (b) DCSI-II.

Table 1. Acoustical parameters of the breast tumour model.

\begin{tabular}{lll}
\hline Tissue & $c\left(\mathrm{~m} \mathrm{~s}^{-1}\right)$ & $\alpha\left(\mathrm{db} \mathrm{m}^{-1}\right)$ \\
\hline Water & 1500 & 0 \\
Fat & 1458 & 41 \\
Glandular & 1519 & 80 \\
Tumour & 1564 & 118 \\
Cyst & 1568 & 10 \\
\hline
\end{tabular}

created by Borup et al [8]. The material contrast model is given by

$$
\chi(\boldsymbol{p})=\frac{c_{0}^{2}}{c^{2}(\boldsymbol{p})}-1+\mathrm{i} \frac{2 \alpha(\boldsymbol{p}) c_{0}}{k_{0} c(\boldsymbol{p})},
$$

where $c$ and $c_{0}$ are the sound speed in the breast tissue and in the embedding water tissue, respectively, while $k_{0}=\omega / c_{0}$. Further, $\alpha$ is the attenuation coefficient in the breast tissue. The true profile is given in figure 1(a). The details of the true profile are given in table 1 .

In the inversion we assumed that the unknown are located within a test domain $D$. The physical size of this test domain is $7.68 \mathrm{~cm} \times 7.68 \mathrm{~cm}$ and is subdivided into $256 \times 256$ sub-domains. First we use a frequency of $250 \mathrm{kHz}$, in which case the size of the test domain is $12.8 \lambda \times 12.8 \lambda$ ( $\lambda$ is the wavelength in water). The circle $S$, where both 64 sources and 64 receivers are located, has a radius of $7.68 \mathrm{~cm}$.

In the results obtained from the Born inversion, shown in figure 1(b), the small regions with tumour tissues are not clearly visible. One should note that these results are obtained using the regularized inversion method in [1]. Figure 1(c) shows the inversion results using DCSI-I. From this figure, it is clear that we get a visibly enhanced reconstruction, which is remarkable considering that it only required small computational overhead compared to 
(a)
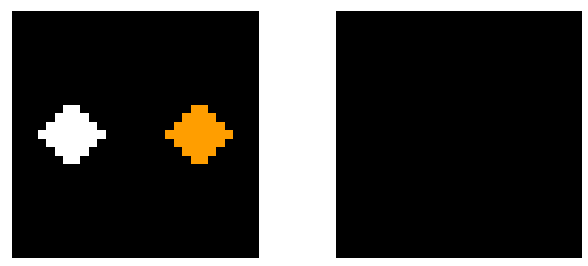

(b)
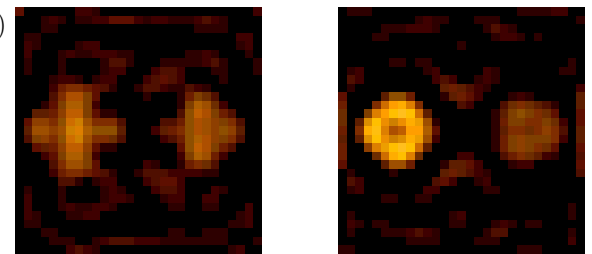

(c)
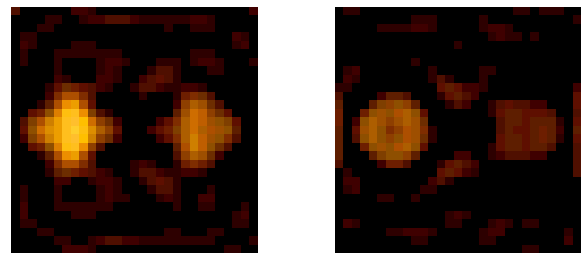

(d)
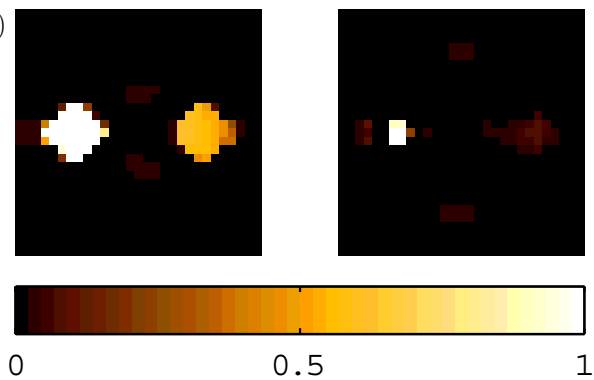

Figure 3. Inversion experiment of the two real homogeneous cylinders; left: real part of $\chi$, right: imaginary part of $\chi$; (a) true profile; (b) Born inversion; (c) DCSI-I and (d) DCSI-II.

the constrained Born inversion [1]. Applying DCSI-II we obtain an even more significant improvement as shown in figure 1(d). This is again remarkable since this method requires only twice the computation time that DCSI-I takes. In this example the inversion process was terminated after 128 iterations for both methods.

In figure 2 we present the inversion results performed at $1 \mathrm{MHz}$. Note that at this frequency the size of the test domain $D$ becomes $51.2 \lambda \times 51.2 \lambda$ and the imaginary part of the contrast $\chi$ is four times smaller than the one at $250 \mathrm{kHz}$. The results of the inversion are shown in figure 2(b). In this case we present only the results obtained using the DCSI-II method. Similar to Natterer [17] and Borup et al [8] we use the results at the lower frequency as the initial estimate for the inversion at the present frequency. We see that the method gives remarkably good results in inverting the real part of the contrast $\chi$. Due to the fact that at this frequency of operation the value of the imaginary part of $\chi$ is very small, the scheme did not produce a reasonable reconstruction of the imaginary part of $\chi$ due to the lack of sensitivity. In this context it is remarked that at this higher frequency of operation the distinction of the tumour location in the imaginary part of the contrast has been lost. 
(a)
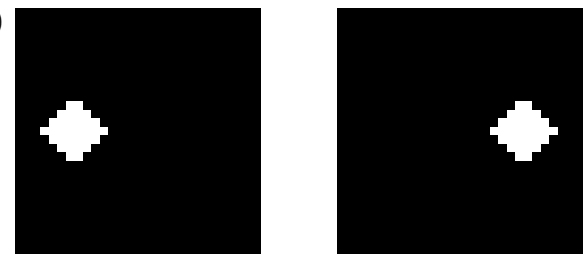

(b)
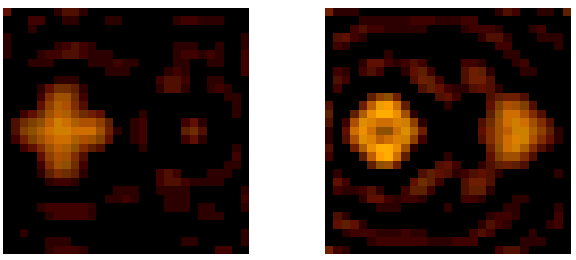

(c)
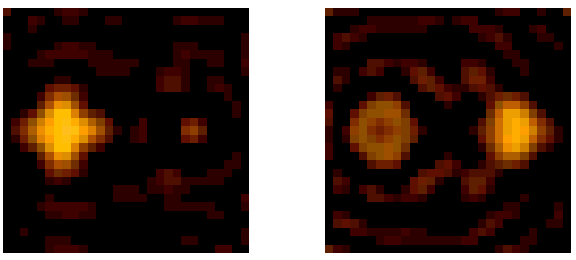

(d)
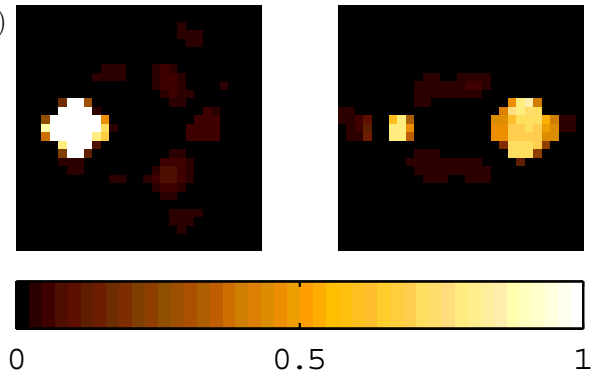

Figure 4. Inversion experiment of the two homogeneous cylinders; left: real part of $\chi$, right: imaginary part of $\chi$; (a) true profile; (b) Born inversion; (c) DCSI-I and (d) DCSI-II.

\subsection{Example 2: real contrast square cylinders}

Next, we will compare the two methods developed in this paper, using a numerical example that was used earlier in [14]. The scattering configuration consists of two distinct square homogeneous cylinders of diameter $3 \lambda / 4$ separated by a distance of $3 \lambda / 4$. The contrast values of the cylinders are real and equal to $\chi=1$ and $\chi=0.5$. The true profile in terms of the material contrast $\chi$ is given in figure $3(\mathrm{a})$. In comparison to the previous example, the contrast is substantially higher.

In carrying out the inversion, we assumed that the scatterers are located within a test domain $D$ with dimensions $3 \lambda \times 3 \lambda$. This test domain is subdivided into $29 \times 29$ sub-domains. The circle $S$, where both 29 sources and 29 receivers are located, has a radius of $3 \lambda$. In this example the inversion process is terminated after 512 iterations. Figure 3(b) shows the results from the Born inversion whereas figure 3(c) shows a significantly improved result from applying DCSI-I. This has been achieved with a minimal extra computational cost. With only double the computational cost of DCSI-I, we achieve an almost perfect reconstruction using 
(a)
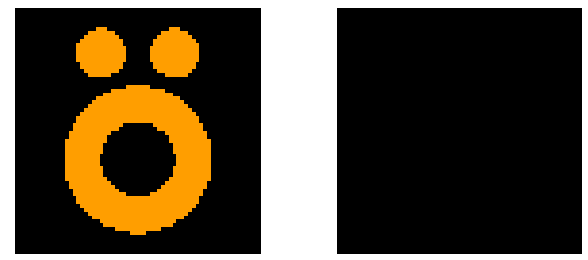

(b)
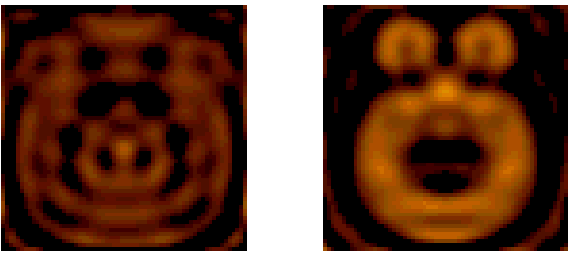

(c)
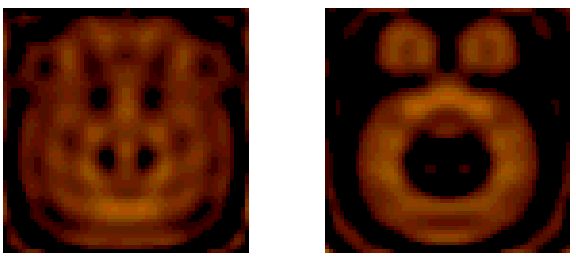

(d)
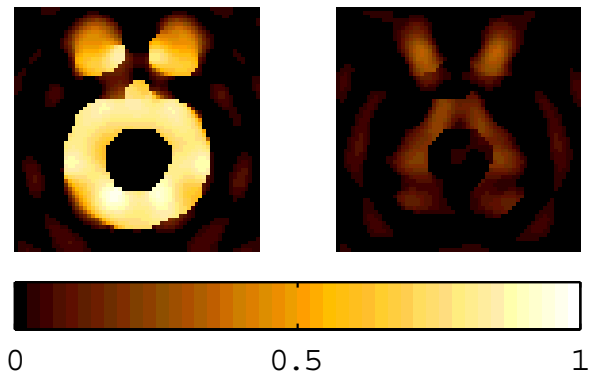

Figure 5. Inversion experiment of the Austria profile; left: real part of $\chi$, right: imaginary part of $\chi$; (a) true profile; (b) Born inversion; (c) DCSI-I and (d) DCSI-II.

DCSI-II. Using DCSI-II, we were able to image the scatterers and accurately identify their location, geometry and material properties.

\subsection{Example 3: complex contrast square cylinders}

As our next experiment, we consider the previous example except that one of the square cylinders has a real-valued contrast and the other has an imaginary-valued contrast. The contrasts of the cylinders have the values $\chi=1$ and $\chi=i$. The true profile is given in figure 4(a). The inversion uses the same measurement setup and the discretization grid as in the previous example. Similar observations as in the first example are made.

\subsection{Example 4: 'Austria' profile}

In our fourth example, a number of single objects are contained in a test domain with sidelength $2 \mathrm{~m}$. These objects consist of two discs and one ring. The discs of radius $0.2 \mathrm{~m}$ are 
(a)
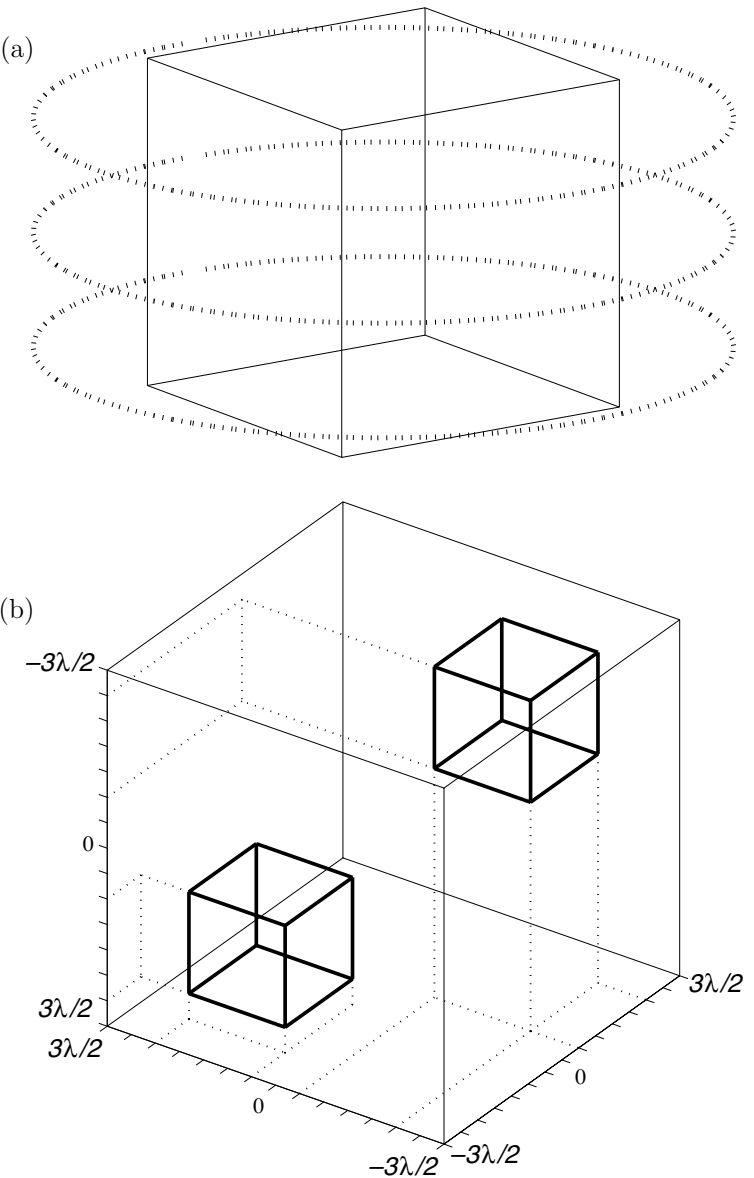

Figure 6. The measurement setup (a) and the configuration (b) of the three-dimensional experiment.

centred at $(0.3,0.6) \mathrm{m}$ and $(-0.3,0.6) \mathrm{m}$. The ring has an outer radius of $0.6 \mathrm{~m}$ and an inner radius of $0.3 \mathrm{~m}$, and is centred at $(0,0.2) \mathrm{m}$. The objects have electromagnetic contrast of $\chi=0.5$. This image is referred to as the 'Österreich' profile by Belkebir and Tijhuis [4]. In their inversion, they used a distorted Born method together with a 'marching-on-in frequency' technique from 100, 200, 300 to $400 \mathrm{MHz}$. Then the wavelengths are $\lambda=3,1.5,1.0$ and $0.75 \mathrm{~m}$. For each frequency, the data were treated separately. The initial guess corresponds to the result of the last iteration of the previously treated frequency. A similar frequency-hopping technique has been applied by Litman et al [16], but using a controlled evolution of a level set for binary objects. They used 64 sources and 65 receivers on a circle of radius $3 \mathrm{~m}$ centred at $(0,0)$, while the test domain was discretized into $30 \times 30$ cells.

We observed that the methods developed in this paper yield favourable reconstruction results using only a single frequency. Here, we present the results using only the data at the highest frequency, i.e., at $400 \mathrm{MHz}$. This is a difficult inversion to consider since at high frequencies, the traditional Born approximation deteriorates in performance. We discretize the test domain into $63 \times 63$ cells and we consider 48 source/receiver stations. The inversion process was terminated after 512 iterations. Despite the reduced set of data, the inversion results show that the quality of reconstruction has not been compromised. The real and 
(a)
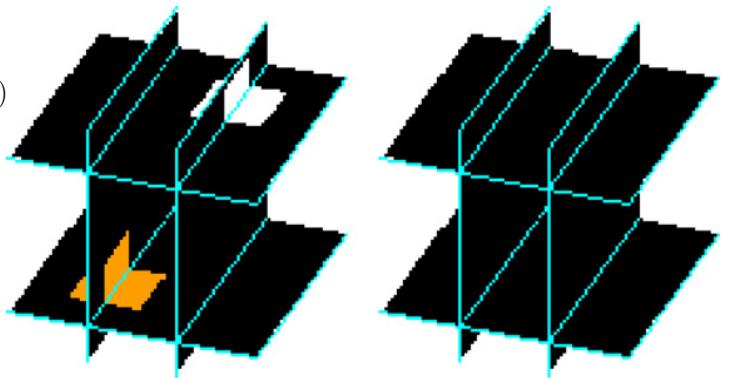

(b)
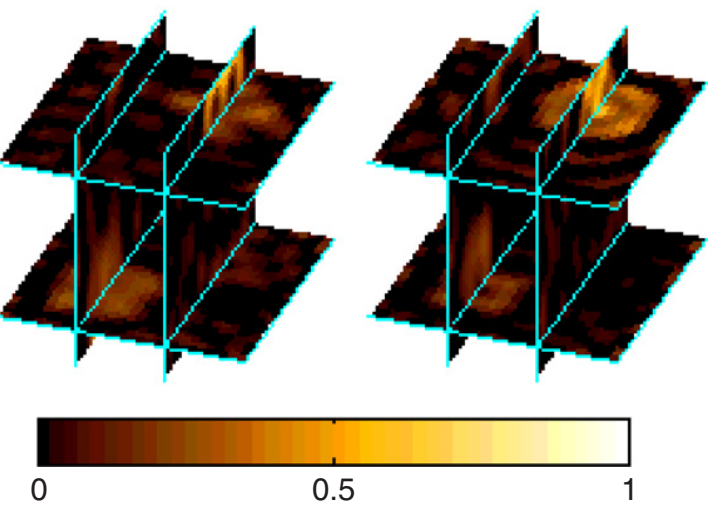

Figure 7. Three-dimensional inversion experiment; left: real part of $\chi$, right: imaginary part of $\chi$; (a) true profile and (b) Born inversion.

imaginary parts of the original profile are presented in figure 5(a). Figure 5(b) shows the results of the Born inversion where, surprisingly, the image of the scatterer appears in the imaginary part of the inverted contrast function rather than in the real part as one would have expected. Method DCSI-I hardly provides any improvements whereas DCSI-II yields a well-defined image of the scatterer with a clear delineation of its various elements. However, the amplitude of the contrast is slightly overestimated. This is caused by the fact that in this example we have a fairly complicated object with a relatively high contrast value. For lower frequencies, we observe that DCSI-I becomes more effective, but with a lower resolution.

\section{Three-dimensional numerical examples}

As a final test, we consider the (scalar) inversion of a three-dimensional configuration shown in figure 6(b). The two scatterers are cubic in shape with a dimension $\lambda$ each. The scatterers have real-valued contrasts and are embedded in a homogeneous background medium. The top scatterer has a contrast value of $\chi=1$, and the bottom scatterer has a value of $\chi=0.5$.

The measurement domain $S$ was chosen to be three rings surrounding the test domain and located at $z=-\lambda, 0, \lambda$, see figure $6(\mathrm{a})$. The incident fields are generated by a total of 30 point sources. Each ring has ten point sources distributed uniformly on a circle of radius $3 \lambda$. Located on the same ring, there are 30 point receivers. The data are collected at all 90 point receivers for each of the 30 point sources. Hence the total number of data points is $90 \times 30=2700$. In the inversion, we divided the test domain $D$, of dimension $3 \lambda \times 3 \lambda \times 3 \lambda$, into $30 \times 30 \times 30$ sub-cubes, resulting in 27000 complex unknowns to be inverted for, a factor of 10 more than the number of data points. 
(a)
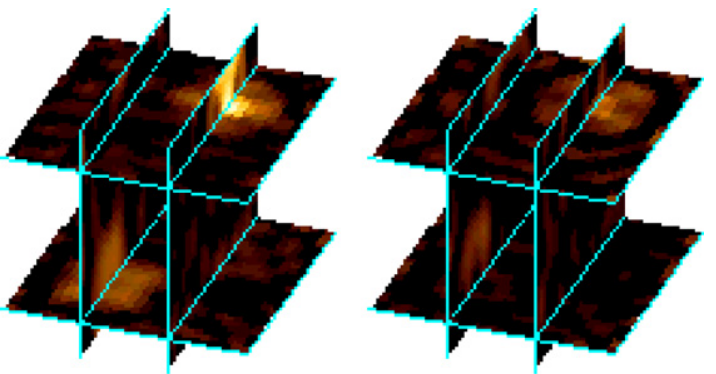

(b)
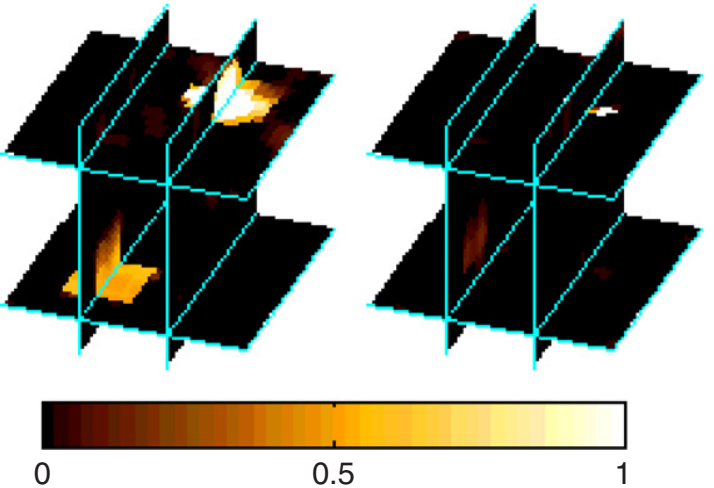

Figure 8. Three-dimensional inversion experiment; left: real part of $\chi$, right: imaginary part of $\chi$; (a) DCSI-I and (b) DCSI-II.

The cross-sectional profile of the scattering configuration is shown in figure 7(a). The inversion results after 1024 iterations using Born, DCSI-I and DCSI-II are shown in figures 7(b), 8(a) and 8(b), respectively. In these figures, the left-hand-side plots contain four slices of the real part of the contrast while the right-hand side contains the imaginary part. The slices are located at the intersection of the scatterers with the planes $x_{1}= \pm \lambda$ and $x_{3}= \pm \lambda$. Examining the real parts of the profile, we observe that the Born inversion provides at best a satisfactory guess on the location of the scatterers. The reconstruction improves with the use of DCSI-I and improves significantly with DCSI-II, see figures 8(a) and (b), respectively. With DCSI-II, the shape, location and amplitude of the contrast of the two objects are well reconstructed.

\section{Conclusions}

In this paper we presented two inverse scattering methods that approximately recast the full nonlinear inversion into a number of linear inversions. The two methods are computationally efficient - as efficient as the constrained born inversion but provide reconstruction results that are far superior and are almost comparable in quality to the ones obtained using the full nonlinear iterative inversion. The computational cost of the methods presented in this paper is only a fraction of the computational cost of the full nonlinear inversion using the contrast source inversion method [26].

The linear inversion steps follow what is referred to as the source-type integral equation approach [12] in which the contrast sources are inverted for in the first step from the linear data equation. Once the contrast sources have been determined, the total wave fields follow from 
a direct application of the object equation. In the third and final step, the contrast function is estimated from either the constitutive relation (DCSI-I) or from solving the data equation, this time in terms of the contrast profile (DCSI-II). The latter approach takes into account higher order scattering effects, which explains the significant improvement in the inversion.

What makes such approaches feasible is the introduction of a diagonalization approximation of the full contrast operator (similar to the concept in [11]). This allows the source-type integral equation axpproach with its three linear steps to be implemented in a robust manner, in which the non-uniqueness encountered in the reconstruction of the contrast sources in the first step is alleviated by the imposition of the diagonal form constraint on the contrast operator.

Two additional enabling methods were introduced to guarantee the robustness and convergence of the above approach. The first method is introduced in the implementation of the first step (of inverting the contrast sources) where we have applied the contrast source inversion of [1]. The second enabling method is introduced in the implementation of the third step (of inverting the contrast profile) where we have applied the multiplicative regularized inversion of [1].

Future research will be directed to extend the present approaches to the full vectorial electromagnetic case. In this case, several diagonalizations of the tensorial contrast operator will be explored. Obviously, multi-component data will be needed for the inversion.

\section{References}

[1] Abubakar A, van den Berg P M and Semenov S 2004 A robust iterative method for Born inversion IEEE Trans. Geosci. Remote Sens. 42 342-54

[2] Abubakar A, van den Berg P M and Mallorqui J J 2002 Imaging of biomedical data using a multiplicative regularized contrast source inversion method IEEE Trans. Microw. Theor. Technol. 50 1761-71

[3] Bao G and Li P 2004 Inverse medium scattering for three-dimensional time harmonic Maxwell equations Inverse Problems 20 L1-7

[4] Belkebir K and Tijhuis A G 1996 Using multiple frequency information in the iterative solution of a twodimensional non-linear inverse problem Proc. PIERS 96: Progress in Electromagnetic Research Symposium (Innsbruck, Austria) p 353

[5] Bleistein N and Cohen J K 1977 Nonuniqueness in the inverse source problem in acoustics and electromagnetics J. Math. Phys. 18 194-201

[6] Born M 1933 Optik (New York: Springer)

[7] Born M and Wolf E 1980 Principles of Optics (New York: Pergamon)

[8] Borup D T, Johnson S A, Kim W W and Berggren M J 1992 Nonperturbative diffraction tomography via Gauss-Newton iteration applied to the scattering integral equation Ultrason. Imaging 14 69-85

[9] Colton D, Coyle J and Monk P 2000 Recent developments in inverse acoustic scattering theory SIAM Rev. 42 369-414

[10] Habashy T M and Mittra R 1987 On some inverse problems in electromagnetics J. Electromagn. Waves Appl. 1 25-58

[11] Habashy T M, Groom R W and Spies B 1993 Beyond the Born and Rytov approximations: a nonlinear approach to electromagnetic scattering J. Geophys. Res. 98 1759-75

[12] Habashy T M, Oristaglio M L and de Hoop A T 1994 Simultaneous nonlinear reconstruction of two-dimensional permittivity and conductivity Radio Sci. 29 1101-18

[13] Habashy T M and Aria Abubakar A 2004 A general framework for constraint minimization for the inversion of electromagnetic measurements Prog. Electromagn. Res. 46 265-312

[14] Kleinman R E and van den Berg P M 1993 An extended range modified gradient technique for profile inversion Radio Sci. 28 877-84

[15] Lesselier D and Duchene B 1996 Wavefield inversion of objects in stratified environments. From backpropagation schemes to full solution Rev. Radio Sci. 1993-1996 (New York: Oxford University Press) pp 235-68

[16] Litman A, Lesselier D and Santosa F 1998 Reconstruction of a two-dimensional binary obstacle by controlled evolution of a level-set Inverse Problems 14 685-706 
[17] Natterer F 1997 An initial value approach to the inverse Helmholtz problem at fixed frequency Inverse Problems in Medical Imaging and Nondestructive Testing ed H W Engl, A K Louis and W Rundell (New York: Springer) pp 159-67

[18] Natterer F 2004 An error bound for the Born approximation Inverse Problems 20 447-52

[19] Nieto-Vesperinas M 1991 Scattering and Diffraction in Physical Optics (New York: Wiley)

[20] Oristaglio M 1985 Accuracy of the Born and Rytov approximations for reflection and refraction at a plane interface J. Opt. Soc. Am. A 2 1987-93

[21] Sabatier P C 2000 Past and future of Inverse Problems J. Math. Phys. 41 4082-124

[22] Bulyshev A E, Souvorov A E, Semenov S Yu, Posukh V G and Sizov Y E 2004 Three-dimensional vector microwave tomography: theory and computational experiments Inverse Problems 20 1239-59

[23] Torres-Verdin C and Habashy T M 1994 Rapid 2.5-dimensional forward modeling and inversion via a new nonlinear scattering approximation Radio Sci. 29 1051-79

[24] Torres-Verdin C and Habashy T M 1995 A two-step linear inversion of of two-dimensional electrical conductivity IEEE Trans. Antennas Propag. 43 405-15

[25] Torres-Verdin C and Habashy T M 2001 Rapid numerical simulation of axisymmetric single-well induction data using the extended Born approximation Radio Sci. 36 1287-306

[26] van den Berg P M and Kleinman R E 1997 A contrast source inversion method Inverse Problems 13 1607-20

[27] van den Berg P M 1981 Iterative computational techniques in Scattering based upon the integrated square error criterion IEEE Trans. Antennas Propag. 32 1063-71

[28] van den Berg P M 2002 Nonlinear scalar inverse scattering: algorithms and applications Scattering: Scattering and Inverse Scattering in Pure and Applied Science ed R Pike and P Sabatier (London: Academic) chapter 3 